\title{
Influencia de la adición del $\mathrm{BaCO}_{3}$ sobre la hidratación del cemento portland
}

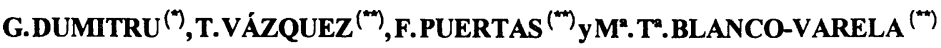 \\ (") University of Craiova, Targu-Jiu, RUMANIA \\ (“) Instituto de Ciencias de la Construcción Eduardo Torroja (CSIC)
}

ESPAÑA

Fecha de aceptación: 26-I-99

\begin{abstract}
RESUMEN
Se ha estudiado el efecto de la incorporación de $\mathrm{BaCO}_{3}$ en la hidratación del cemento portland. Como resultado del estudio se ha concluido que la presencia de $\mathrm{BaCO}_{3}$ en los cementos hace que éstos requieran una mayor cantidad de agua de amasado. Sin embargo, las resistencias mecánicas no se ven negativamente afectadas, ya que se produce la formación de carboaluminatos por reacción química entre el carbonato de bario y el $\mathrm{C}_{3} \mathrm{~A}$ del clinker. El $\mathrm{BaCO}_{3}$ manifiesta un efecto retardador en los tiempos de fraguado, tanto en presencia como en ausencia de yeso. $\mathrm{El} \mathrm{BaCO}_{3}$ reacciona con el yeso formando $\mathrm{BaSO}_{4}$ sal de muy elevada estabilidad. La formación de esta sal de bario inhibe la formación de ettringita en las pastas. Finalmente, indicar que la presencia de $\mathrm{BaCO}_{3}$ en los cementos asegura que éstos no experimenten procesos expansivos cuando se encuentren en medios sulfáticos externos o internos, ya que el carbonato interaccionará con dichos sulfatos formando BaSO, e impidiendo la formación de ettringita expansiva.
\end{abstract}

\section{SUMMARY}

The effect of incorporation of $\mathrm{BaCO}_{3}$ in portland cement hydration has been studied. From this study it is inferred that presence of $\mathrm{BaCO}_{3}$ in cements makes them require a higher quantity of mixing water. However, mechanical resistances do not seem negatively affected, for a formation of carboaluminates by chemical reaction between the barium carbonate and the $\mathrm{C}_{3} \mathrm{~A}$ of the clinker appears. The $\mathrm{BaCO}_{3}$ reveals a retardant effect on the setting time, in presence of gypsum as much as in its absence. The $\mathrm{BaCO}_{3}$ with gypsum reacts as $\mathrm{BaSO}_{4}$, a salt of a very high stability. The formation of this barium salt inhibits the formation of ettringite in pastes. Finally, the presence of $\mathrm{BaCO}_{3}$ in cements ensures that no expansive process will occur in sulphatic means either external or internal, for carbonate is going to react with these sulphates and form $\mathrm{BaSO}_{4}$, preventing thus the formation of expansive ettringite.

\section{INTRODUCCIÓN}

En un estudio previo (1) se ha comprobado que el $\mathrm{BaCO}_{3}$ retarda la reacción de hidratación del $\mathrm{C}_{3} \mathrm{~A}$ de modo similar al producido en presencia del $\mathrm{CaCO}_{3}(2-3)$. En ese mismo trabajo se ha puesto en evidencia que la composición mineralógica de los productos de hidratación del $\mathrm{C}_{3} \mathrm{~A}$ en presencia de $\mathrm{BaCO}_{3}$, tienen una peculiaridad específica, que se manifiesta por la formación de un hemicarboaluminato, de fórmula $\mathrm{C}_{8} \mathrm{~A}_{2} \mathrm{cH}_{24}$. En la composición de este hemicarboaluminato ha sido identificado un $6 \%$ de $\mathrm{Ba}$. En presencia de yeso, el $\mathrm{BaCO}_{3}$ acelera la reacción de hidratación del $\mathrm{C}_{3} \mathrm{~A}$. $\mathrm{El} \mathrm{BaCO}_{3}$ inhibe la formación de ettringita, ya que precipita la sal insoluble $\mathrm{BaSO}_{4}$.

En el presente trabajo el objetivo planteado ha sido conocer la influencia que puede ejercer el $\mathrm{BaCO}_{3}$ en el proceso de hidratación del cemento portland.

\section{PARTE EXPERIMENTAL}

\subsection{Metodología}

Se partió de dos clínkeres de cemento portland, uno de ellos bajo en $\mathrm{C}_{3} \mathrm{~A}$ (denominado $\mathrm{B}$ ) y otro alto en $\mathrm{C}_{3} \mathrm{~A}$ 
(denominado $\mathrm{N}$ ). La composición química y el cálculo potencial de Bogue de los citados clínkeres se presenta en la Tabla I.

Los clínkeres $\mathrm{B}$ y $\mathrm{N}$ fueron molidos separadamente hasta alcanzar unas finuras correspondientes a unos Blaines de $3.980 \mathrm{~cm}^{2} / \mathrm{g}$ y $3.940 \mathrm{~cm}^{2} / \mathrm{g}$, respectivamente. A partir de estos clínkeres molidos se prepararon los siguientes 4 tipos de cementos:

1) cementos $\mathrm{B}$ con yeso y adiciones de $\mathrm{BaCO}_{3}$.

2) cementos $\mathrm{N}$ con yeso y adiciones de $\mathrm{BaCO}_{3}$.

3) cementos $\mathrm{B}$ sin yeso y con adiciones de $\mathrm{BaCO}_{3}$.

4) cementos $\mathrm{N}$ sin yeso y con adiciones de $\mathrm{BaCO}_{3}$.

La cantidad de yeso adicionada, de una pureza del $95,60 \%$, se fijó teniendo en cuenta el contenido de $\mathrm{C}_{3} \mathrm{~A}$ y de sulfatos alcalinos en los clínkeres. La proporción de $\mathrm{BaCO}_{3}$ adicionada varió entre $0-25 \%$ en peso de cemento.
Sobre esos cementos se realizaron los siguientes ensayos y determinaciones:

a) Contenido de agua necesaria para obtener una pasta de cemento de consistencia normal.

b) Tiempos de fraguado.

c) Resistencias mecánicas en probetas normalizadas de mortero $1 / 3$.

d) Análisis mineralógico por Difracción de Rayos $\mathrm{X}$ (DRX).

\section{RESULTADOS}

3.1. Contenido de agua necesaria para obtener una pasta de cemento de consistencia normal

Este contenido de agua ha sido determinado de acuerdo a la Norma UNE-80-102-88 (5) y al Pliego RC-97 (6). Los resultados obtenidos se presentan en la Tabla II

TABLA I

Composición de química de los clínkeres. Cálculo potencial de Bogue

\begin{tabular}{|c|c|c|}
\hline $\begin{array}{c}\text { DETERMINACIONES } \\
\text { (\% en masa) }\end{array}$ & CLINKKER B & CLÍNKER N \\
\hline Pérdida al fuego & 0,25 & 0,61 \\
\hline Residuo insoluble & 0,19 & 0,24 \\
\hline $\mathrm{SiO}_{2}$ & 20,81 & 20,27 \\
\hline $\mathrm{Al}_{2} \mathrm{O}_{3}$ & 4,03 & 6,07 \\
\hline $\mathrm{Fe}_{0} \mathrm{O}_{3}$ & 5,40 & 3,32 \\
\hline $\mathrm{CaO}$ & 65,96 & 66,27 \\
\hline $\mathrm{MgO}$ & 0,90 & 0,96 \\
\hline $\mathrm{SO}_{3}$ & 1,15 & 0,90 \\
\hline $\mathrm{CaO}$ libre & 0,85 & 1,20 \\
\hline $\mathrm{Na}_{2} \mathrm{O}$ & 0,20 & 0,22 \\
\hline $\mathrm{K}_{2} \mathrm{O}$ & 0,80 & 0,85 \\
\hline LSF & 0,992 & 0,983 \\
\hline Ms & 2,20 & 2,20 \\
\hline $\mathrm{MF}$ & 0,75 & 1,83 \\
\hline $\mathrm{C}_{3} \mathrm{~S}$ & 74,00 & 66,90 \\
\hline $\mathrm{C}_{2} \mathrm{~S}$ & 4,50 & 9,18 \\
\hline $\mathrm{C}_{3} \mathrm{~A}$ & 3,00 & 11,48 \\
\hline $\mathrm{C}_{4} \mathrm{AF}$ & 16,41 & 10,12 \\
\hline
\end{tabular}


TABLA II

Relaciones agua/cemento

\begin{tabular}{|c|c|c|}
\hline $\begin{array}{l}\text { NOMBRE } \\
\text { CEMENTO }\end{array}$ & $\begin{array}{c}\text { AGUA DE } \\
\text { CONSISTENCIA NORMAL } \\
(\% \text { en masa })\end{array}$ & $\begin{array}{c}\text { RELACION } \\
\text { AGUA/CEMENTO }\end{array}$ \\
\hline $\mathrm{B}-0$ & 28,0 & 0,280 \\
\hline B -5 & 28,5 & 0,285 \\
\hline $\mathrm{B}-10$ & 29,0 & 0,290 \\
\hline$B-15$ & 29,3 & 0,293 \\
\hline$B-20$ & 29,9 & 0,299 \\
\hline $\mathrm{B}-25$ & 30,1 & 0,301 \\
\hline $\mathrm{N}-0$ & 30,0 & 0,300 \\
\hline $\mathrm{N}-5$ & 30,6 & 0,306 \\
\hline$N-10$ & 31,0 & 0,310 \\
\hline $\mathrm{N}-15$ & 32,0 & 0,320 \\
\hline$N-20$ & 32,5 & 0,325 \\
\hline $\mathrm{N}-25$ & 33,1 & 0,331 \\
\hline B - 0 SIN YESO & 30,5 & 0,305 \\
\hline B - 5 SIN YESO & 31,5 & 0,315 \\
\hline B - 10 SIN YESO & 32,0 & 0,320 \\
\hline B - 15 SIN YESO & 33,0 & 0,330 \\
\hline B - 20 SIN YESO & 33,3 & 0,333 \\
\hline B - 25 SIN YESO & 33,6 & 0,336 \\
\hline $\mathrm{N}$ - O SIN YESO & 31,7 & 0,317 \\
\hline $\mathrm{N}$ - 5 SIN YESO & 32,0 & 0,320 \\
\hline $\mathrm{N}$ - 10 SIN YESO & 32,6 & 0,326 \\
\hline $\mathrm{N}$ - 15 SIN YESO & 33,1 & 0,331 \\
\hline $\mathrm{N}-20$ SIN YESO & 33,3 & 0,333 \\
\hline $\mathrm{N}$ - 25 SIN YESO & 33,7 & 0,337 \\
\hline
\end{tabular}

\subsection{Tiempos de fraguado}

En la Tabla III se muestran los tiempos de inicio y final de fraguado obtenidos en los distintos tipos de cementos estudiados (5).

\subsection{Resistencias mecánicas}

Los valores experimentales medios obtenidos en la de- terminación de las resistencias mecánicas a 7 y 28 días, aparecen recogidos en la Tabla IV.

\subsection{Análisis por DRX}

En las Tablas V a VIII se presentan los resultados obtenidos por DRX sobre las pastas estudiadas. Se da una valoración semicuantitativa de composición de fases. 
TABLA III

Tiempos de fraguado de las pastas

\begin{tabular}{|c|c|c|c|}
\hline $\begin{array}{l}\text { NOMBRE } \\
\text { CEMENTO }\end{array}$ & $\begin{array}{l}\text { PRINCIPIO DEL } \\
\text { FRAGUADO } \\
\text { (minutos) }\end{array}$ & $\begin{array}{l}\text { FINAL DEL } \\
\text { FRAGUADO } \\
\text { (minutos) }\end{array}$ & $\begin{array}{l}\text { TIEMPO DEL } \\
\text { FRAGUADO } \\
\text { (minutos) }\end{array}$ \\
\hline$B-0$ & $1 \mathrm{~h}-19$ & in -39 & oh -20 \\
\hline B- 5 & $2 h-14$ & $3 h-14$ & $1 \mathrm{~h}-00$ \\
\hline$B-10$ & $3 h-04$ & $4 h-14$ & $1 \mathrm{~h}-10$ \\
\hline B -15 & $3 h-16$ & $4 h-48$ & $1 \mathrm{~h}-32$ \\
\hline B -20 & $3 h-28$ & $4 h-59$ & $1 \mathrm{~h}-31$ \\
\hline $8-25$ & $3 h-38$ & $5 n-28$ & $1 h-30$ \\
\hline$N-O$ & $1 \mathrm{~h}-04$ & $1 h-29$ & On -25 \\
\hline$N-5$ & $2 h-54$ & $3 h-54$ & $1 \mathrm{~h}-00$ \\
\hline$N-10$ & $3 h-24$ & $4 h-32$ & $1 \mathrm{~h} \cdot 08$ \\
\hline$N-15$ & $3 h-34$ & $4 h \cdot 46$ & $1 \mathrm{n}-12$ \\
\hline$N-20$ & $3 h-52$ & $4 h-59$ & $1 n \cdot 07$ \\
\hline$N-25$ & $3 h-55$ & $5 \mathrm{~h}-17$ & $1 \mathrm{~h}-22$ \\
\hline B - O SIN YESO & $\mathrm{Oh} \cdot 44$ & $2 \mathrm{~h}-00$ & On 14 \\
\hline B - 5 SIN YESO & $1 \mathrm{~h}-15$ & $2 h \cdot 00$ & $\mathrm{Oh}-45$ \\
\hline B - 10 SIN YESO & $1 \mathrm{~h} \cdot 36$ & $2 h-41$ & $1 \mathrm{~h}-05$ \\
\hline B - 15 SIN YESO & $1 \mathrm{~h} \cdot 48$ & $2 h \cdot 45$ & $1 \mathrm{~h}-03$ \\
\hline B - 20 SIN YESO & $1 \mathrm{~h}-55$ & $5 h-55$ & $1 \mathrm{~h}-10$ \\
\hline B - 25 SIN YESO & $1 \mathrm{~h}-57$ & $3 h-18$ & $1 \mathrm{~h}-21 \mathrm{~m}$ \\
\hline$N$ - O SIN YESO & $\mathrm{Oh}-36$ & Oh -55 & Oh -19 \\
\hline$N-5$ SIN YESO & Oh -42 & Oh - 59 & Oh -17 \\
\hline$N-10$ SIN YESO & $1 \mathrm{~h} \cdot 19$ & $1 \mathrm{~h}-58$ & Oh - 39 \\
\hline$N-15$ SIN YESO & $1 \mathrm{~h} \cdot 28$ & $2 h-39$ & $1 \mathrm{~h} \cdot 11$ \\
\hline$N-20$ SIN YESO & $1 \mathrm{~h}-35$ & $2 h-52$ & $1 \mathrm{~h}-17$ \\
\hline$N-25$ SIN YESO & $1 \mathrm{~h} \cdot 42$ & $2 h-59$ & $1 \mathrm{~h}-18$ \\
\hline
\end{tabular}

TABLA IV

Valores experimentales medios de las resistencias mecánicas

\begin{tabular}{|c|c|c|c|c|}
\hline \multirow[t]{2}{*}{$\begin{array}{l}\text { NOMBRE } \\
\text { CEMENTO }\end{array}$} & \multicolumn{2}{|c|}{$\begin{array}{l}\text { R. FLEXOTRACCION (MPa) } \\
\text { VENCIMIENTO }\end{array}$} & \multicolumn{2}{|c|}{$\begin{array}{l}\text { R. COMPRESION (MPa) } \\
\text { VENCIMIENTO }\end{array}$} \\
\hline & 7 DIAAS & 28 DIAS & 7 DIAS & 28 DIAAS \\
\hline$B-0$ & 6,98 & 7,42 & 47,90 & 51,70 \\
\hline$B-5$ & 6,79 & 7,39 & 46,32 & 52,56 \\
\hline$B-10$ & 6,82 & 7,47 & 46,49 & 51.74 \\
\hline B -15 & 6,73 & 7,59 & 46,06 & 49,52 \\
\hline B- 25 & 6,70 & 7.55 & 45,87 & 49,97 \\
\hline $\mathrm{N}-\mathrm{O}$ & 6,72 & 7,25 & 42,62 & 53,90 \\
\hline$N-5$ & 6,44 & 7,56 & 42,68 & 52,76 \\
\hline$N-10$ & 7,11 & 7,69 & 44,15 & 53,36 \\
\hline$N-15$ & 6,82 & 7,47 & 42,70 & 52.20 \\
\hline B - 15 SIN YESO & 6,62 & 7,30 & 36,00 & 49,45 \\
\hline B - 25 SIN YESO & 6.30 & 6,52 & 36,23 & 43,66 \\
\hline
\end{tabular}


TABLA V

Cementos tipo B

Fases identificadas por DRX de las pastas hidratadas a los 28 días

\begin{tabular}{|c|c|c|c|}
\hline FASES & B-0 & B-10 & B-20 \\
\hline Alita & + & + & + \\
\hline $\mathrm{C}_{3} \mathrm{~A}$ & + & 0 & - \\
\hline $\mathrm{Fase} \mathrm{Fe}$ & + & + & + \\
\hline $\mathrm{BaCO}_{3}$ & - & ++ & ++ \\
\hline $\mathrm{BaSO}_{4}$ & - & + & + \\
\hline $\mathrm{Ca}_{2}\left(\mathrm{OH}_{2}\right.$ & ++ & ++ & ++ \\
\hline Ettringita $_{2}$ & - & - & - \\
\hline $\mathrm{C}_{4} \mathrm{AcH}_{11}$ & - & 0 & 0 \\
\hline
\end{tabular}

TABLA VII

Cementos tipo B sin yeso

Fases identificadas por DRX en las pastas hidratadas a los 28 días

\begin{tabular}{|c|c|c|c|}
\hline FASES & $\mathrm{B}-0-\sin \mathrm{y}$ & $\mathrm{B}-10-\sin \mathrm{y}$ & $\mathrm{B}-20 \sin \mathrm{y}$ \\
\hline Alita & ++ & ++ & + \\
\hline $\mathrm{C}_{3} \mathrm{~A}$ & + & 0 & 0 \\
\hline $\mathrm{Fase} \mathrm{Fe}$ & + & + & + \\
\hline $\mathrm{BaCO}_{3}$ & - & ++ & ++ \\
\hline $\mathrm{BaSO}_{4}$ & - & + & + \\
\hline $\mathrm{Ca}_{3}(\mathrm{OH})_{2}$ & ++ & + & + \\
\hline $\mathrm{Ettringita}_{\text {trita }}$ & - & - & - \\
\hline $\mathrm{C}_{4} \mathrm{AcH}_{41}$ & - & 0 & 0 \\
\hline
\end{tabular}

++++ abundante (mayoritario)

+ poco

+++ mucha

0 trazas

++ moderado

\section{DISCUSIÓN}

La presencia de $\mathrm{BaCO}_{3}$ en las pastas incrementa los requerimientos de agua necesarios para conseguir mezclas de igual consistencia (ver Tabla II). Sin embargo, este mayor contenido de agua en las pastas no tiene un efecto negativo sobre las resistencias mecánicas, tanto a flexotracción como a compresión (Tabla III). A partir de los resultados obtenidos tras el análisis mineralógico realizado por DRX, lo anteriormente expuesto se puede justificar por la formación de carboaluminatos en las mezclas, como consecuencia de la reacción del $\mathrm{C}_{3} \mathrm{~A}$ del cemento anhidro con el carbonato de bario. Es conocido en bibliografia que la formación de monocarboaluminatos va asociada a incrementos en las resistencias mecánicas (7). Este incremento podría compensar la esperada pérdida de resistencias que tendrían las pastas con altos contenidos en $\mathrm{BaCO}_{3}$, ya que éstas tienen menor cantidad de silicatos cálcicos hidratados.
TABLA VI

\section{Cementos tipo $\mathrm{N}$}

Fases identificadas por DRX en las pastas hidratadas a los 28 días

\begin{tabular}{|c|c|c|c|}
\hline FASES & $\mathrm{N}-0$ & $\mathrm{~N}-10$ & $\mathrm{~N}-20$ \\
\hline Alita & ++ & + & + \\
\hline $\mathrm{C}_{3} \mathrm{~A}$ & + & - & - \\
\hline $\mathrm{Fase} \mathrm{Fe}_{\mathrm{a}}$ & - & - & - \\
\hline $\mathrm{BaCO}_{3}$ & - & + & +++ \\
\hline $\mathrm{BaSO}_{4}$ & - & + & + \\
\hline $\mathrm{Ca}_{2}\left(\mathrm{OH}_{2}\right.$ & ++ & ++ & ++ \\
\hline $\mathrm{Ettringita}$ & + & - & - \\
\hline $\mathrm{C}_{4} \mathrm{AcH}_{11}$ & - & + & + \\
\hline
\end{tabular}

TABLA VIII

Cementos tipo $\mathrm{N}$ sin yeso

Fases identificadas por DRX en las pastas hidratadas a los 28 días

\begin{tabular}{|c|c|c|c|}
\hline FASES & $\mathrm{N}-0-\sin \mathrm{y}$ & $\mathrm{N}-10-\sin \mathrm{y}$ & $\mathrm{N}-20-\sin \mathrm{y}$ \\
\hline Alita & + & + & + \\
\hline $\mathrm{C}_{3} \mathrm{~A}$ & 0 & - & - \\
\hline $\mathrm{Fase} \mathrm{Fe}$ & - & - & - \\
\hline $\mathrm{BaCO}_{1}$ & - & ++ & +++ \\
\hline $\mathrm{BaSO}_{4}$ & - & + & + \\
\hline $\mathrm{Ca}_{4}(\mathrm{OH})_{2}$ & +++ & +++ & ++ \\
\hline Ettringita & - & - & - \\
\hline $\mathrm{C}_{4} \mathrm{AcH}_{11}$ & - & + & + \\
\hline
\end{tabular}

Como era de esperar, la cantidad de monocarboaluminatos en las pastas es mayor en aquéllas que proceden de cementos con altos contenidos en $\mathrm{C}_{3} \mathrm{~A}$.

$\mathrm{El} \mathrm{BaCO}_{3}$ también afecta a los tiempos de fraguado de las pastas (Tabla III), tanto en los cementos con alto contenido en $\mathrm{C}_{3} \mathrm{~A}$ (cementos $\mathrm{N}$ ) como aquéllos con bajo (cementos B). Los tiempos de inicio y el final del fraguado se alargan al incrementarse la proporción de carbonato de bario en las mezclas. En ausencia de yeso este fenómeno también es observado, aunque el efecto es menos significativo. El alargamiento en los tiempos de fraguado por la presencia de carbonato de bario se debe, por una parte, al mayor contenido de agua en las pastas $y$, por otra, al efecto retardador del $\mathrm{BaCO}_{3}$.

El $\mathrm{BaCO}_{3}$ reacciona con el yeso presente en las mezclas formando $\mathrm{BaSO}_{4}$ (baritina), sal de elevadísima estabilidad e insolubilidad. La formación de este sulfato de bario 
inhibe la formación de ettringita en las pastas. Este fenómeno ya había sido observado al estudiar el efecto del carbonato de bario sobre la hidratación del $\mathrm{C}_{3} \mathrm{~A}(1)$.

\section{CONCLUSIONES}

Las principales conclusiones que se pueden extraer del presente trabajo son:

i) La presencia de $\mathrm{BaCO}_{3}$ en cementos hace que éstos requieran una mayor cantidad de agua de amasado. Sin embargo, las resistencias mecánicas no se ven negativamente afectadas, ya que se produce la formación de carboaluminatos por reacción química entre el carbonato de bario y el $\mathrm{C}_{3} \mathrm{~A}$ del clínker. Esos carboaluminatos contribuyen a un incremento en las resistencias.

ii) $\mathrm{El} \mathrm{BaCO}_{3}$ manifiesta un efecto retardador en los tiempos de fraguado, tanto en presencia como en ausencia de yeso.

iii) $\mathrm{El} \mathrm{BaCO}_{3}$ reacciona con el yeso formando $\mathrm{BaSO}_{4}$, sal de muy elevada estabilidad. La formación de esta sal de bario inhibe la formación de ettringita en las pastas.

iv) La presencia de $\mathrm{BaCO}_{3}$ en cementos asegura que éstos no experimenten procesos expansivos cuando se encuentren en medios sulfáticos externos o incluso cuando la fuente de sulfatos sea interna, ya que el carbonato interaccionará con dichos sulfatos formando $\mathrm{BaSO}_{4} \mathrm{e}$ impidiendo la formación de ettringita expansiva.

\section{AGRADECIMIENTOS}

Los autores, y particularmente el Profesor Dr. Gheorghe Dumitru en la Universidad "Constantin Brancusi" de la ciudad Targu Jiu, en Rumania, quisieran expresar su agradecimiento a la Dirección General de Investigación Científica y Técnica (DGICYT) del Ministerio de Educación y Ciencia de España como Organismo financiador debido al cual se ha realizado esta investigación, así como al C.S.I.C. y a la Dirección del I.E.T.C. C. por la confianza depositada.

\section{BIBLIOGRAFÍA}

(1) G. DUMITRU, T. VÁZQUEZ, F. PUERTAS, Ma .T. BLANCO VARELA: $10^{\text {th }}$ Intern. Congr.of the Chem. of Cem. (Gothenburg), Vol3,5pp(1997).

(2) S. RAMACHANDRAN: $8^{\text {th }}$ Intern. Congr. of the Chem. of Cem.(Riode Janeiro), Vol 6, pp.178-182.(1986).

(3) J.BENSTED: Word Cement Technology, 14, No. 10, pp. $395-$ 406(1980).

(4) C. VERNET: $8^{\text {th }}$ Intern. Congr. of the Chem. of Cem. (Rio de Janeiro), Vol.3, pp. 70-74 (1986).

(5) Norma UNE 80-102-88(1988).

(6) Instrucción para recepción de cementos RC 97. Ministerio de Fomento. España(1997).

(7) J. SOROKA, N. STERN: Cement and Concrete Research, Vol.3,pp. 367-376(1976).

\title{
Publicación del Instituto Eduardo Torroja - CSIC
}

\author{
Número monográfico de INFORMES
}

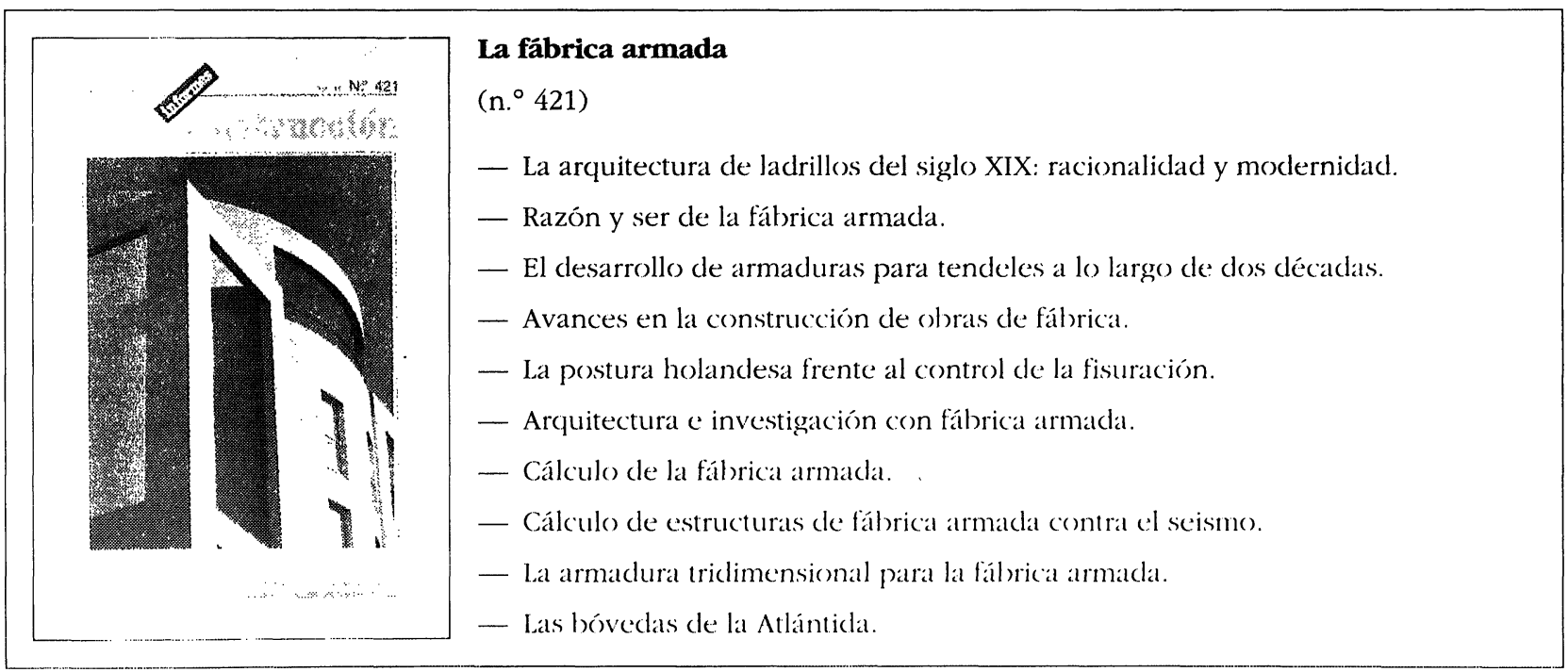

